\title{
P047. Paroxysmal episodic hemicrania in a child. A complex differential diagnosis
}

\author{
Roberto Frusciante ${ }^{1 *}$, Alessandro Capuano ${ }^{1}$, Catello Vollono², Federico Vigevano ${ }^{1}$, Samuela Tarantino1, \\ Massimiliano Valeriani ${ }^{1,3}$ \\ From Abstracts from the 1st Joint ANIRCEF-SISC Congress \\ Rome, Italy. 29-31 October 2015
}

\section{Background}

Headache is a common disease in children. Differential diagnosis of primary headaches in children is challenging due to some peculiar features of headache at that age. We describe a case in a child who presented with headache resembling characteristics of migraine without aura, paroxysmal hemicrania and cluster headache.

\section{Methods and results}

An 11-year-old boy referred to our Headache Centre suffering from headache for the past 5 years. Headache characteristics were: frontal pain, constricting in quality and excruciating pain intensity, at times vomiting. Duration of attacks was referred ranging from 20 to $40 \mathrm{~min}$ utes, headache occurred daily during the last 2 months. Attacks recurred many times daily. Attacks of headache occurred in periods lasting from 1 to 2 months, separated by pain-free periods lasting 1 month. During the headache attacks, the child presented eyelid oedema and nasal congestion. Personal medical history was negative. Familial history was positive for migraine without aura (paternal aunt). General and neurological examination, including fundus oculi, were normal. MRI scan resulted normal. Previous prophylaxis with pizotifen, amitriptyline, verapamil, topiramate and prednisone were ineffective, instead indomethacin was effective.

\section{Discussion}

In children, the characteristics of headache often overlap between different forms. Cluster headache is a rare form and very few cases in pediatric age have been described [1]. Our patient presented headache attacks with autonomic activation (eyelid oedema and nasal congestion), that usually rule out the diagnosis of migraine attacks. On the contrary, these features are thought to be specific of TACs. Furthermore, the clustering of attacks, more than one per day, the ineffectiveness of different prophylactic therapies, and the complete efficacy of therapeutic doses of indomethacin, support the diagnosis of paroxysmal episodic hemicrania in our patient.

Written informed consent to publication was obtained from the patient(s).

\section{Authors' details}

'Headache Centre, Bambino Gesù Children's Hospital, Rome, Italy. 2Department of Geriatrics, Neurosciences \& Orthopedics, Unit of Neurophysiopathology and Sleep Medicine, Catholic University, Policlinico Agostino Gemelli, Rome, Italy. ${ }^{3}$ Centre for Sensory-Motor Interaction, Aalborg University, Aalborg, Denmark.

Published: 28 September 2015

\section{Reference}

1. Tarantino S, Vollono C, Capuano A, Vigevano F, Valeriani M: Chronic paroxysmal hemicrania in paediatric age: report of two cases. $J$ Headache Pain 2011, 12(2):263-7.

\section{doi:10.1186/1129-2377-16-S1-A100}

Cite this article as: Frusciante et al:: P047. Paroxysmal episodic hemicrania in a child. A complex differential diagnosis. The Journal of Headache and Pain 2015 16(Suppl 1):A100. 